\title{
Vie de sainte Elisabeth de Hongrie par Nicolas Bozon,
}

\section{Introduction.}

La vie de sainte Elisabeth de Hongrie par Nicolas Bozon, frère mineur, n'est connue jusqu'à présent que par un court extrait de Francisque Michel, Rapports all Ministere, t. I. pp. 258-70 et par l'introduction de M. Paul Meyer à l'édition des Contes moralisés, S. A. T. F. 1889. En publiant cette vie nous croyons contribuer à la connaissance des œuvres de Bozon.

\section{L'auteur.}

Nicolas Bozon nous est connu, grâce à sa fécondité littéraire. L'abbé De la Rue dans ses Essais historiques sur les Bardes, les Jongleurs et les Trouvères normands et anglo-normands (Caen, 1834) a voulu voir en lui le neveu et secrétaire du pape Adrien IV, mort en 1181. Francisque Michel a attribué également les vies du ms. au Brit. Nus. à l'abbé Bozon de l'abbaye du Bec, mort le 23 jnin 1176. Wright, Biogr. Brit. t. II (1846), p. 331, a contesté l'hypothèse de $\mathrm{De}$ la Rue, mais il a cru aussi que le poète vivait au XIII ${ }^{e}$ siècle. N. Paul Meyer, Contes moralisis, 1889, pp. I-III, a corrigé cette erreur que la vie de sainte Elisabeth rend évidente. B. était originaire du nord de l'Angleterre et il a composé ses Contes après 1320 . M. P. W. Harry n'a pas contribué beaucoup à la connaissance de l'auteur par son A comparative Study of the Aesopic fable in Nicole Bozon, 1905. Une foule d'études ont suivi ia publication des Contes et M. Harry en a dressé la liste. M. Maurice Hewlett A Medineval Popular Preacher, The Nineteenth Century, t. XXVIII (18go) pp. 470-477 a essayé de préciser un peu nos renseignements sur Buzon. Sâ jatrie est Nottingham, Derby ou Staffordshire; le nom de Bozon appartenait à une famille de Norfolk. Il a vécu entre 1320 et 1350 . Il a possédé un savoir vaste pour son temps, car il connait la Bible, Pline, Dioscoride, Aristote, les Gestes d'Alexandre et de Charles, les Gesta Romanorum, le noble clerke Avicenne, le bon clerke Basilius, Isidore, S. Bernard, s. Grégoire, Barlaam et Josaphat, Bède, S. Ambroise.

L.époque où Bozon a vécu est la fin du treizième et le commencement du quatorzième siècle. MI. P. Meyer dit dans l'Introduction des Contes moralisés (p. II): „Au $\$ 144$, il est question de l'évôque John d'Alderby, qui occupa le siège épiscopal de Lincoln de 1300 à 1320. La manière dont ist est parlé de ce personnage donne à 
penser qu'il était mort lorsque Bozon écrivait. Les contes auraient donc été rédigés après 1320 ." La vie de s. Elisabeth nous semble de la fin du treizième siècle. Nous en allons donner deux preuves: la langue de l'auteur est l'anglo-normand de la fin du XIII ${ }^{e}$ siècle, puis l'un des mss. qui nous conserve le poème fut terminé vers I 304.

Les poésies de Bozon sont presque toutes inédites. Th. Wright, Jubinal, M M. Meyer et Baker ont publiés quelques pièces. M. Paul Meyer en a donné des extraits en analysant le ms. Cheltenham, Bibl. Phillipps 8336 (Ch) dans la Romania t. XIII (1884) p. 496 et 554 et Francisque-Michel dans ses Rapports au Ministre t. I, pp. 258 -70. Voici la liste avec l'indication des mss. d'après les recherches de M. P. Meyer:

I. Le Char d'Orgueil; mss.: ${ }^{0}$ Ch. ff. 66-74; 20 Cambridge, Un. Libr. $\mathrm{Gg}$ 6. $28 \mathrm{ff}$. I-3; $3^{0}$ Londres, B. M. Old roy. $8 \mathrm{E}$ XVII fol. $188 \mathrm{v} ; 4^{0}$ Oxford, Bibl. Bodl., Bodley 425, fol. 94.

2. De la bonti des femmes; ms. Ch. ff. $93-95$ v.

3. La femme comparke à la pie; mss.: $1^{0}$ Ch. fol. $75 ; 2^{0}$ Londres, B. M. Harl. 2253 , fol. 112.

4. Poime allegorique sur la Passion; mss.: I $^{0}$ Ch. ff. 58-70; 20 Londres, B. M. Cotton Jul. A V.

5. Traite de "denaturesce"; ms. Ch. fol. 49 v.

6. Sermons en vers; ms $C h$. ff. $80-83$ (nos 25-3 I de l'analysc).

7. Poime sur l'annonciation; ms. Ch. fol. 75 v.

8. Priere d la Vierge; ms. Ch. ff. $50 \mathrm{v}-5 \mathrm{Ir}$.

9. Paraphrase de l'Ave Maria; ms. Ch. fol. 50.

10. Proverbes de bon enseignement; mss. ${ }^{0}$ Oxford, Bibl. Bodl., Bodley 425; $2^{0}$ Londres, B. M. Old roy. $8 \mathrm{E} \mathrm{XVII;} 3^{0}$ Oxford, Bibl. Bodl., Selden supra 74.

I1. Vie de s. Lucie; ms. Londres, Brit. Mus., Cotton Dom. XI

(B) ff. $9 \mathrm{r}-92$.

12. Vie de Marie Madeleine; ms. $B$ ff. $92-95$.

13. Vie de s. Marguerite; ms. $B$ ff. 95-97.

14. Vie de s. Marthe; ms. $B$ ff. $97-99$.

15. Vie de s. Elisabeth; mss. 10 B ff. 99-101 ; $^{0}$ Weibeck, Bibl. Portl. I $C_{1}(W)$ ff. $1-4$.

16. Vie de s. Christine; ms. $B$ ff. $101 \mathrm{v}-102 \mathrm{v}$.

17. Vie de s. Julienne; ms. B ff. $102 \mathrm{v}-103 \mathrm{v}$.

18. Vie de s. Agnes; ms. $B$ ff. $103 \mathrm{v}-105 \mathrm{v}$.

19. Vie de s. Agathe; ms. $B$ ff. $105 \mathrm{v}-107$.

20. Vie de s. Panuce; ms. $W$ ff. $4-5$ v. (Cf. Romania XXXVill p. $418-424)$.

21. Vie de s. Paul l'Ermite ms. $W$ ff. 6-8. (Cf. Mod. L. Rev. VII, 1909).

\section{Les manuscrits.}

La vie de s. Elisabeth par Bozon se trouve dans deux mss.

I. Francisque-Michel a donné une description et une analyse du ms. de Londres, Brit. Mus., Cotton Dom. XI dans ses Rapports 
en 1838. C'est un volume in $4^{0}$ qui contient 182 feuillets sur vélin, écrits à deux colonnes dont chacune a 42 lignes. Il y a deux feuillets de garde dont le premier contient la Table et le dernier une charte. L'écriture est du $\mathrm{XIV}^{\mathrm{e}}$ siècle, mais on y remarque trois mains: la première jusqu'au fol. $106 \mathrm{v}$, la deuxième jusqu'au fol. 154 .

Le ms. renferme outre les vies de saintes énumérées parmi les wuvres de B. (nos II-19):

1. La vie de s. Elmont par Denis Piramus (ff. I-24), publiée par Th. Arnold dans les Memorials of St. Elmund Abbey (London, 1892, Rolls series II, p. 137) et par Lord J. Hervey dans la Corolla S. Edmunili (London, 1907 p. 224). Cf. Hist. litt. XXXIII, p. 346.

2. La vie de s. Thomas de Cantorbiry par Garnier de PontSaint-Maxence ff. 25-43 publiée d'après un ms. de Wolfenbuttel par J. Bekker dans les Abhandlungen der köniylichen Akadeniie zu Berlin (1838, p. 25) et d'après un ms. de Paris par Hippeau (Paris, I559). Ct. Hist. litt. t. XXIII, p. j67 et t. XXXIII, p. 376/377.

3. La Genèse de N. D. s. Marie ff. $43-80$. Cf. Hist. litt. t. XXXIII, p. 366.

4. L'assomption de N. D. sainle Marie ff. $80-87$. Cf. Hist. litt. t. XXXIII, p. 366 . p. 356.

5. Traduction de l'Évangile ff. $87-9$ I. Cf. Hist. litt. t. XXXIII,

6-I 4. Cf. II, II-I9.

15. Liber formularum de noto electionis etc. in usum monasterii B. Mariae Helluni in urbe Rothomago etc. ff. 107-155.

16. De exequiis et sepultura Fmerii decimi octavi abbatis monasterii B. Mariae de Becco anno 1304, et de electione et benedictione successoris Gilleberti et gestis ipsius ff. 155-180.

La troisième main a écrit le dernier texte latin qui nous semble être contemporain du texte français copié par la première. L'écrit est une notice d'annales (cf. Hist. litt. t. XXXII, p. 211 ) et ce genre ne permet pas de placer sa rédaction beaucoup après l'événement. Nous possédons donc une copic des poèmes de Bozon faite vers 1304 et la composition doit être placée avant cette date.

2. Une copie plus rícente se trouve à Welbeck,' Bibl. du duc de Portland I C I ff. I-4. C'est le seul ms. français de cette bibliothèque suivant le Cahulogute par Arthur Strong (cf. Romania XXXII, p. 637). M. Paul Meyer l'avait utilisé en composant son article pour l'Hist litt. t. XXXIII, p. 334 n. 2, et M. A.-T. Baker vient de publier de ce ms. la Vie de saint Panuce ff. $4-5 \mathrm{v}$ qu'il attribue à Bozon et la Vie de s. Paul (Mod. L. Rev. VII, 1909).

1 Nous adressons tous nos remerciments à M. R. Goulding, bibliothècaire de S. A. le duc de Portland à Welbeck, qui a bien voulu déposer le ms. au département des mss. du British Muséum et à M. A. R. Dryhurst adjoint au même musée qui a bien voulu se charger du ms. jusqu'à ce que nous ayons terminé notre étude. 
On trouvera ailleurs l'analyse du ms. avec des extraits, nous devons nous borner à quelques remarques. Le volume a 264 feuillets sur vélin (deux feuillets de plus sont en blanc), écrit à deux colonnes dont chacune a 32 lignes. Les rubriques sont en rouge, une seule initiale en bleu; quelques unes sont ornées de miniatures. La plupart des vies de saintes renfermées dans ce ms. sont encore inédites et ne furent pas mentionées avant l'article cité dans l'Hist. litt.

Je vais suivre le texte du ms. $B$ dans l'édition suivante, mais je donne les variantes de ms. $W$ en bas de la page. Pour ne pas encombrer les notes, je négligerai les variations d'orthographe: $1 . B$ $\mathrm{y}: W \mathrm{i}, 2$. $h$ initial fréquent dans $W ; 3.5$ devant les consonnes manque dans $W ; 4 . B$ ay : $W$ ey; 5. u, o: $W$ ou; 6 . a, o nasal : $W$ aun, oun. Je donnerai le poème tel qu'il est sorti de la main du scribe qui parlait le même dialecte anglo-normand que le poète. L'étude de la langue et de la versification va nous renseigner sur ce point.

\section{La langue.}

L'introduction des Contes moralisés (Paris, I889) pp. Lll-LXVI nous renseigne sur l'expansion et la période de domination de l'anglo-normand en soulignant quelques faits remarquables chez Bozon. Mais l'étude complète de la langue de Bozon doit être remise jusqu'à ce que nous ayons une édition satisfaisante de toutes ses poésies. La publication des textes anglo-normands a été assez riche dans le dernier quart du $19^{e}$ siècle et M. John E. Matzke vient de dresser une liste d'éditions dans l'introduction (p. XIX. XX.) des CEuvres de Simund de Freine (Paris, 1909). Nous ne voulons pas entreprendre une comparaison des formes du langage avec d'autres poésies de Bozon ou de ses contemporains, mais nous devons relever quelques traits marquants qui justifieront l'attribution du poème à Bozon et permettront d'en tirer des conclusions pour la date de la composition.

\section{Phonétique.}

1. Bozon fait rimer lat. $a=$ fr. e et lat. $a=$ fr. ie avec: $e$ libre comme tous les auteurs anglo-normands: aierger her 1, mangi santé 59, pitíe meseisée 89, muster esquier 109, pé areillée 205.

2. L'auteur ne sépare pas les infinitifs en are de ceux en ere: voler visiter 37, poer prier 139, aler aver 319, purchacer aver 395. 3. Une seule rime prouve le mélange de $o$ et $u$ : prodomme custume 177 .

4. En général les rimes en $e i$ et en $a i$ sont pures: vey rey 391, fay mai (= lat. me) I39, desray may 2 I I.

Il n'y a qu'une seule rime en -estre qui est pure: destre estre 381 .

5. La diphtongue $u i$ est souvent réduite à $u$ : sut nut 123 , nut fut 207, apparut nut 275, 291, escut dedut 335. La réduction 
de $u i$ à $i$ se présente aussi quelques fois: issi li 107, tolli li 185 , suffri li 199, issi nuli 357.

6. La diphtongue ue se trouve dans le mot quer: prier quer 21 , auter quer 137,267 , quer targer 163.

7. Lat. $o, \mathrm{u}=$ agln. ou se trouve à la rime avec ou: jour creatour 27, jour seignour 197, 217, seignour demour 225, socour creatour 293, oure seignour 399; oure demasure 343.

8. Les nasales $a+n$ et $e+n$ suivies d'une consonne ne riment qu'avec les mêmes sons et cette distinction caractérise bien les textes anglo-normands: avent vent 9, bien rien 49, gent largement 95, prent dedeynement 117 , comandement attent 239, meyntenant lusant $24 \mathrm{I}$.

Dans le ms. $B$ la seule rime servaunte aunte $6_{3}$ indique le changement de an en aun devant une consonne. Pareilles rimes sont fréquentes dans le ms. W: mangé saunté 59 , ploraunt pussaunt 23I, meyntenaunt lusaunt $24 \mathrm{I}$ et vivaunt 273, devaunt regardaunt 239, encountraunt en faunt 273, enfaunt lusaunz 383. En acceptant l'opinion de Stürzinger (Ortographia Gallica, Heilbronn, I 884, XXXIX) que ce phénomène appartient à la deuxième moitié du XIII ${ }^{e}$ siècle, on pourrait de cela préciser la date relative des deux textes: $B$ conserve les traces d'un dialecte plus ancien et l'auteur semble ne pas avoir connu cet usage.

\section{Consonnes.}

9. Ie changement du lat. $1=u$ après $a, e, 0$ s'est déjà accompli dans notre texte: haut chaut 347 . Les mots avec $l$ mouillé se trouvent avec d'autres sons d̀ la rime: merveyléz cntremelléz 245, merveylá chaunged $35 \mathrm{I}$.

10. Les sons $n$ et $m$ ne sont pas toujours séparés à la rime: peissoun houm (W) et homme (B) 53, mais: peissoun mesoun 65; an dame 93.

11. Le $t$ est resté dans les préterits en it: sentit espirit 16j, mit dit 141, ienit escondit 219 et attendit 223, nasquit ait 375; dans ceux en ut: merciout pout 29, dut fut 75, out priout 103, crut morut 321 , escut dedut 335, out donout 397, pout alout 403 .

12. Les sons $s$ et $z$ se confondent dans le ms.: hus puz $5 \mathrm{I}$, mais plusieurs rimes les séparent: leprous dous 69 , gent vestimenz 255, enfanz (corr. enfant) lusanz 383 et l'auteur semble les avoir distingués. Le son $s$ devant $t$ de la terminaison est tombé: enquist languit 43 et vit 275 , cessat estat $15 \mathrm{I}$.

I 3. Les labiales sont tombés devant $s$ : jolifs aris I II.

\section{Morphologie.}

Déclinaison: La flexion se trouve dans l'état de décadence. L's de flexion du nominatif au singulier est tombé: angel 295; mais a l'accusatif du pluriel on le retrouve: les povres genz 18, petiz ioches 48 . Les masculins de la troisième déclinaison prouvent la confusion des cas: nom. sire 211 , sires 222, seignour 217 ; pro- 
domme 214 . Le cas régime n'est pas toujours exprimé par la préposition: le rey 7 , seignour 210 . Le vocatif n'a pas d's: Dieu 83 .

L'article masc. au cas sujet est au singulier $l i$ (écrit ly) $32 \mathrm{I}$, mais auss le 77, 362, 382; l'article féminin: la 334; au pluriel les $69,95,400$.

Les pronoms personnels ont les formes suivantes: jeo 139, nus 2, vus 127, il 183, ele I15, li 22, la 183, 393, cls 20, les 58 .

Le pronom relatif présente la forme ke pour le nominatif aussi bien que pour l'accusatif: $k e 67,84,102$; au nom. on trouve aussi $k i: 44$.

Conjugaison. Les formes du verbe ne donnent pas lieu à beaucoup de remarques. Bozon distingue les imparfaits de la première conjugaison qui sont en oue de ceux des autres conjugaisons qui sont en eie: pout alout 403, merciout pout 29, deveit diseit 147, assingneit aveylleit 203.

Aveir se trouve: aveit 336, out 103, 142, ut 288, eez 280;

Esteir se présente: estei 364, esteit 7, 229, 244, 301, esteient 411 , seez I16, serrez 117, serreit 64.

\section{La versification.}

M. Matzke (l. c. p. XLIII) vient de résumer les problìmes de la versification anglo-normande en suivant Gnerlich, Bemerkungen ïber den Virsbau der Anglonormannen, Stra/sburg, i 88o. Il y a encore beaucoup de points à éclaircir. D'autre part les poésies de Bozon sont en grande partie inédites et elles ne nous sont restées que dans un seul ms.; il est donc difficile, et il le sera toujours, d'avoir une idée exacte de sa manière de versification. Nous allons relever les traits caractéristiques du poème publié.

Il est écrit en couplets de vers octosyllabiques; la loi de M. Paul Meyer pour les couplets est valable presque sans exception. Quant au nombre des syllabes il $y$ a beaucoup de vers irréguliers que j'ai indiqués comme corrompus. Sur 414 vers du poème 140 sont trop longs ou trop courts. On peut en corriger quelques-uns, mais la plupart de ces vers incorrects sont dus à l'auteur. Il usait largement des licences acceptées par les poètes anglo-normands et c'est sur la base de ces principes que je grouperai les faits caractéristiques pour la détermination du nombre des syllabes.

I. B. fait élider l'e atone final même quand il n'est pas suivi d'une voyelle:

a) après une voyelle: folie 118 ;

b) après une liquide: le 14, 16, 131, 202, 403; ele 42, 95, $115,174,187,191,225,286,357,394$; bele 56, 62; uncle 64; mule 66, 394; sele 66; merveyle 244; parole 303; damoysele 351; novele 385; autre 142; tere 154; fere 208; povre 71, 189; dire 84, 356; estre 130, 316; mestre 172; mere 275; oure 290; riere 342; quere 388; ne 64, 188, 397; une 108, 267, 270, 330, 331; bone 131; dame 188, $215,283,313,413$; homme 274, 298; custume 100; 
c) aussi après une autre consonne: de 48, 105, 202, 256, 303, $337,385,414$; ke 104, 147, 246, 274, 412; unke 53; servante 62, 330; viande 75; quite 295; joyouse 337; servisce 14; abstinence 73; srace 166; face 381 ; comence 344 ; fuse 189.

Un assez grand nombre de vers peuvent être corrigés par cette élision qui est suffisamment établie dans d'autres textes. Il est à remarquer que Bozon l'emploie même après une sourde suivie d'une liquide: autre, povre, estre, mestre.

2. La terminaison -ent s'élide après une voyelle dans les mots suivants: esteient 4 I I, porteient 4 I 2.

3. L' $\epsilon$ atone entre consonnes est souvent syncopé: overer 32 , avercit 46, ayderai 139, lezer 346, aunceles 187, nouveles 188, angel 235, caneles 257 , sustenance 333 , sodeynement 118,363 , joyousement I 94 ; dans la particule: entremellez 246; espirit 4 I 2 est trisyllabique.

4. L'hiatus au milieu du mot peut subsister presque avec toutes les voyelles: poez 130, doüte 80, voüt 316 , encoüntra 331 , esquier 1 10; mais creatour 294 est bisyllabique.

5. La contraction de la voyelle finale avec l'initiale n'est pas permise: ou unke 53; mais elle doit être supposée: e a 388 .

6. La syllabe initiale peut tomber: sa entent 386; c'est le cas avec la particule de: demora 330, dehors 19 ; avec re: regardey 359; celi est monosyllabique.

7. L'e final atone n'est pas toujours élidé devant une voyelle. M. Rydberg, Zur Geschichle des französischen o (Leipzig 1897) groupe les faits en hiatus logique, métrique, grammatical, analogique et dialectal. Il est difficile quelquefois de ranger les phénomènes dans l'un ou l'autre groupe. On trouve dans notre texte l'hiatus logique: ke assingnée 208, noble attifure 236; métrique: le ose 84 ; grammatical: jeo ay 160, la (le) apparut 275.

En général l'e dans la même position s'élide, même dans le pronom ke: k'ele $71, k \circ j 330$.

Fn admettant toutes ces licences de la versification anglonormande, il reste encore un certain nombre de vers fautifs. D'après le ms. $W$ on doit corriger 4 vers: $66,81,131,182$. J'ai employé la paranthèse pour marquer la syllabe ajoutée et les crochets pour celle qui est retranchée.

En considérant les principes énumérés ci-dessus 83 vers deviennent réguliers. Il en reste encore 53 qui sont soit trop longs, soit trop courts établis par l'accord des mss. Les vers trop longs sont les suivants: $87,122,139,1.77,171,179,185,210,254,258$, $278,367,369,371,386,390$. Les vers trop courts: 22, 109, 157, $401 ; 33,36,40,51,55,57,74,79,88,112,129,138,151$, I 52 , $184,192,193,201,211,237,242,245,250,259,271,308,318$, $322,323,328,345,375,406$.

Je n'ai corrigé ni les uns ni les autres, car toutes les corrections proposées restent incertaines jusqu'à la découverte d'un nouveau ms. 
Les bibliothèques de la Grande Bretagne ne sonst pas encore dépouillées et surtout on n'a pas fait de recherches spéciales pour Bozon. La conclusion de notre étude est que Bozon a admis des vers trop longs, mais réguliers d'après les principes de la versification anglo-normande et des vers trop courts dont la raison d'adinission est jusqu'ici inconnue.

\section{La source latine.}

La question de savoir, quelle est la vie latine que Bozon a traduite, ne laisse pas de présenter certaine difficulté. Parmi les quatre vies en vers français sur s. Elisabeth, Rutebeuf traduit le livret qui contient les dépositions des quatre servantes', la vie de Bruxelles celle de Thierry d'Apolda, le poème de Robert de Cambligneul est en partie la copie de la vie de Bruxelles et il se tient aussi à la déposition des servantes. Mais Bozon traite sa matière avec beaucoup de liberté, comme M. Paul Meyer l'a remarqué pour la vie de saint Agnès.

Nous désignerons parmi les écrits publiés ceux qui pouvaient donner des renseignements à notre auteur. Avant de le faire, nous donnerons en résumé les faits racontés dans le poème. vv. 1 -6.

I. Dieu s'est manifesté dans une dame noble, s. Elisabeth

2. Elle est la fille d'un roi, elle est bien sage et bonne vv. 7-12.

3. Dans sa jeunesse elle sert Dieu, elle visite les pauvres et leur distribue ses biens vv. 13-22.

4. Elle est mariée au duc de Thuringe et dans l'état de mariage elle continue de servir dieu et son mari l'approuve vv. 23-30.

5. Au temps de la disette elle fait construire un hôpital ou elle nourrit les pauvres vv. $3 \mathfrak{I}-42$.

6. Premier miracle: un malade demande des poissons, Elisabeth va les chercher $d$ un puits où jamais on n'en a vu; elle remporte les poissons grâce auquel le malade est guéri vv. 43-66.

7. Sa dévotion se fait voir par les soins extrêmes à pratiquer les ceuvres de pitié; elle s'impose l'humilité et l'abstinence. Sa prière a des effets miraculeux vv. 67-106.

8. Deuxième miracle: un chevalier outrecuidant se présente devant Elisabeth; elle lui conseille plus d'humilité et le fait prier par crainte de sa mort. Le chevalier sent telle angoisse qu'il crie au secours et se fait moine vv. 107-164.

9. Elisabeth se soumet à l'obéissance de maître Conrad qui lui impose de grandes privations vv. 165-1 84 .

10. Elisabeth désire l'état de pauvreté vv. 185-196.

11. Une servante l'éveille chaque matin pour aller prier; elle tire une fois le pied du duc qui n'en est pas irrité vv. 197-212.

' Cf. C. Friesland dans $Z R P h$. XIX (1895) p. 375-382 (erreur: Elisabeth "böhmische Prinzessin" malgré les vers 147 et 258 cités). 
1 2. Troisième miracle: le duc veut lui présenter un seigneur; Elisabeth s'excuse car elle n'est pas assez bien mise. ¿̀ sa prière dieu lui envoie des vêtements admirables vv. $213-248$.

13. Elisabeth soigne les malades, elle fatigue son corps et mène une vie dévote vv. 249-266.

14. Quatrième miracle: sa mère morte lui apparaît et demande son secours, car elle doit expier son peché. Elisabeth prie pour sa mère et celle-ci est délivrée de sa peine vv. 267-296.

15. Cinquième miracle: à l'hôpital Elisabeth rend la parole et le mouvement libre à un muet et paralitique vv. 297-316.

16. Le duc va d la croisade et il y meurt vv. 317-322.

17. Elisabeth est exilée par les parents de son mari vv. 323 -328 .

18. Elisabeth s'en va avec une seule servante; une vieille femme, à laquelle elle a fait du bien, refuse de lui céder le pas dans un sentier étroit et Elisabeth tombe dans la boue; elle s'essuie avec joie vv. $329-338$.

19. Sixième miracle: Jésus Christ lui apparaît ce qui la rend joyeuse, mais sa disparition l'attriste vv. $339-372$.

20. Septième miracle: un aveugle-né reçoit la vue par le toucher de s. Elisabeth vv. 373-384.

2 I. Son père veut rappeler sa fille, il lui envoie un messager, mais Elisabeth veut rester en pauvreté; elle distribue tous les biens qu'on lui donne vv. $385-397$.

22. Les ossements du duc sont rapportés et Elisabeth leur rend les derniers honneurs vv. $398-403$.

23. Elisabeth se retire du monde et vit en sainte; à sa mort on entend une douce mélodie qui vient des anges vv. 404-4r4.

Le dossier de s. Elisabeth s'est enrichi avec une rapidité étonnante. On trouve les documents énumėrés dans la Bibliotheca hagingraphica latina pp. $273-277(B H)$ et il y faut ajouter l'édition de quelques écrits publiés par M. Huyskens (1. c. pp. 1 10-266). Bozon semble avoir consulté quelque recueil de miracles dont nous possédons trois rédactions: de $1232(B . H .2)$, de $1233(B . H .4)$, de 1235 (II. p. 243). Pour la vie de s. Elisabeth il pouvait avoir sous les yeux le Libellus de $1235(B . H .6)$ oì le document du procès de canonisation a revêtu la forme de biographie suivant M. Huyskens qui croit avoir retrouvé la rédaction originale de Nicolas (H. p. I 12 ). Mais il y a encore deux vies du treizième siècle: celle de Césaire d'Heisterbach $(C . H$.) dans un ms. de Nördlingen $(B . H .7)$ de 1 236-37 et celle de Thierry d'Apolda (B. H. 9) de 1289-1297 dans plusieurs rédactions. Il y faut ajouter la lettre de Conrad de 1232 qui accompagnait la liste de miracles citée et peut-être deux autres lettres privées sur la mort de s. Elisabeth ( $H$. p. 148) de I 23 I et sur les miracles (H. p. I 50) de I 232.

Bozon donne peu d'importance au faits biographiques; ceux-ci ne servent que pour relier les sept miracles qu'il a encadrés dans son poìme. Ces miracles se trouvent dans les vies à partir du 
Libellus qui servait de source aux écrits postérieurs. Bozon ne semble pas suivre Césaire de Heisterbach, car il accepte l'exil de s. Elisabeth que celui-i ne connaît pas. Il n'a pas connu la vie de Thierry, car il néglige certains faits et noms qu'il aurait relevés, s'il avait consulté l'œuvre du premier biographe de s. Elisabeth.

Le Libellus reste la source principale, mais on doit tenir compte de la lettre de Conrad et quelque liste de guérisons miraculeuses. Nous suivrons l'édition Menckenius, Script. rer. Germ. t. II, pp. 2007 -34 $(M)$ pour le premier, celle de Wyss, Hessisches Urkundenbuch t. I, pp. 25-29 $(W)$ pour le second écrit. Il n'y a que deux miracles $(6,14)$ qu'on ne trouve ni chez l'un ni chez l'autre, mais bien dans la vie de Thierry $(T h)$ qui les a puisés dans une source inconnue. $\mathrm{Au}$ commencement notre auteur abrège beaucoup, il néglige presque entièrement la déposition de Guda et après l'introduction commence par celle d'Isentrude. Cette introduction rappelle pour le style une vie postérieure publiée dans le Catal. Codd. Hag. Bibl. Reg. Bruxel. pp. 398-445 (Boll.). Mais cette forme lapidaire est bien propre à $B$. et les faits mentionnés se trouvent bien dans sa source nommée.

Le tableau suivant fera voir le rapport du poème analysé avec ses sources latines:

\begin{tabular}{|c|c|c|c|c|}
\hline$B$ & $W$ & $M$ & $T / 2$ & Boll. \\
\hline I. & & & & $402,26-29$ \\
\hline 2. & & & $\mathrm{I}, \mathrm{I}$ & $403, I-8$ \\
\hline 3. & & 202 I D & & \\
\hline 4. & 32. $34-35 ; 39-40$ & $2017 \mathrm{D}$ & I, 8; III, 8 & \\
\hline 5 . & $32,30-40 ; 33,15-16$ & $2022 \mathrm{~A}-\mathrm{B}$ & III, 6 & \\
\hline 6. & & & VI, 8 & \\
\hline 7. & & $2014 \mathrm{C}$ & III, II & \\
\hline 8. & & $2024 \mathrm{C}-2025 \mathrm{~A}$ & & \\
\hline 9. & $33,13-15$ & 2014 B & IV, 9 & \\
\hline 10. & $32,40-33,12$ & $2018 \mathrm{C}$ & $v, 8$ & \\
\hline II. & & $2015 \mathrm{C}$ & II, I & \\
\hline 12. & & & II, 8 & \\
\hline 13. & $33,15-20$ & 2023 C-D & & \\
\hline 14. & & & VI, 8 & \\
\hline 15. & & (Th) $2002 \mathrm{~A}-\mathrm{C}$ & (cf. $M$ ) & \\
\hline 16. & & & IV, $\mathrm{x}$ & \\
\hline 17. & & 2019 B & IV, 7 & \\
\hline 18. & & $2019 \mathrm{D}-2020 \mathrm{~A}$ & IV, 8 & \\
\hline 19. & & $2020 \mathrm{C}$ & VIII, I & \\
\hline 20. & & $2033 \mathrm{~A}$ & & \\
\hline 2 I. & & $2028 \mathrm{~B}$ & VI, 6 & \\
\hline 22. & & $2021 \mathrm{C}-\mathrm{D}$ & $\mathrm{V}, 6$ & \\
\hline 23. & & 203 I B-C & VIII, 4 & \\
\hline
\end{tabular}




\section{Text.}

De seinte Elizabeth ceste la vie [fol.g9b.]

La fille le roy de Ungarie.

Novele chose en nostre verger

A nus se mustra avant her:

Une dame de grant noblesce,

4 Fille de rey e grant duchesce,

Seinte Elizabeth est nomée,

Pur li dieus ad overée.

Fille esteit le rey de Ungarye,

8 Sage e curteise e bien nurrie;

$E$ pur ceo ke sovent avent

Ke bounté de nurture vent,

Plus out en li de bounté

$12 \mathrm{Ke}$ ne put estre ore mustré.

Ele comencea mult par tens

En le servisce dieu user son tens;

Tut fut ele mult honurée,

16 Le honour del mound ad poy amée.

Chescun jour ele visita

Les povres genz ke mult ama; [fol.g9c]

De hors le palays les fit venir

20 E de ses biens o els partir,

E sovent les pria de prier

Dieu pur li de bon quer.

Kant ele vint de gregnur age,

24 Son pere la purvit de mariage;

Al duk de Turring fut mariée,

Homme noble e allosée,

Ke suffrit bien nute $e$ jour

$28 \mathrm{Ke}$ ele servit son creatour,

Dunt ele s'en jooit e merciout

Dieu e li que fere le pout.

Lors commencea de enoycer

32 Sa devocion par bien overer;

Pur le amur Jhesu Crist

En pouvre genz myt son delit,

Dunc ele fit un hospital

36 Pur povre genz en un val

Où ele pout a son voler

Chescun jour els visiter.

De un en autre s'en ala,

40 E a chescun demanda

Kubrique: $\mathrm{La}$ vie de seynte Elizabeth. [f. Ia]

I N. rose de $n$. 13 mout 14 deu, $25 \mathrm{Au} 27$ ben 
Quele viande vout manger,

$\mathbf{E}$ memes ele fit atirer.

Un jour avynt ke ele enquist

44 De un malade ki languit

Quele viande desireit,

Ele promit k'il le auereit.

„Dame, dit il, cink ou sis

48 De petiz loches, mes en ceo pais

Ne sount poynt trovez, jeo sey bien."

La dame ne fit autre rien,

Fors s'en va dehors le hus,

52 O une gace, a un puz

$O$ unke loche, ne autre peissoun,

Ne fut trovée de nul homme.

Si reporta en sa mayn

56 Des bele loches la gace pleyn;

De sa mayn les attira

$E$ al malade les dona.

Si tost cum il en out mangé

60 Par dieu resceut pleyn santé. [fol.ggd]

Tel miracle fit dieu pur cele

Ke servante le fut bone e bele;

$\mathrm{Ki}$ ut ore tel servaunte,

64 Ne serreit mester pur uncle ne aunte

Al marché mander pur peissoun,

Nous n'avoun nule tele a mesoun.

Cele ke fut de si grand lyn

68 Sovent se mit a tere enclyn

Pur laver les pez de leprous

$E$ beysa lur pez de quer douze.

Unkes a povre $k$ 'ele encontra

72 Si ele ut prest ke ele ne dona.

Par abstinence son cors demeyne,

Privement mit en peyne;

De chescun viande ke manger dut

76 A vant ke mangeast, certeyne fut

Ke le dener fut bien gaygné

Dunt la viande fut achaté.

S'il venit de merciment

80 Ou pur doute a present

Ou par prey de roberye:

42 le f. $\quad 43$ enquit $\quad 44$ une, 46 ke il 48 petite 49 ben 52 gache 54 nostre houm 55 mey 56 gache 58 au 59 tot, $B$ mangée, $W$ mangé 60 deu, pleyne sauntee $6 \mathrm{I}$ Teu 63 tele 65 pessoun, $f . I c \quad 66 B$ Nus ne aucuns $W$ Nous n'avoun 67 graunt $69 B$ leprouses $W$ leprous 70 playes, 74 myt 75 cheun $76 B$ Quant W Avaunt, 77 bon 8I preye 
Ja ne gustereit en sa vie

Dieu, cum ci ad bele chose

84 Le poy est usé, si dire le ose!

Sovent se mit a baptizer

De povres enfanz pur els lever,

K'ele ut enchesun par tel mester

88 Les enfanz apres ayder.

Tant fut esmue de pitiée,

Kant ele vit nul meseisée,

K'ele ne pout sey retenir

92 Ou de lermer ou de suzpir.

Le jour del cene chescun an

Ne ubblia point la bone dame

K'ele ne lava les porre gent

96 E les regarda largement.

De rien ne fut escoymusée

Fors de pechié venymousé.

A muster ala mult matins

I00 De custume pur oyer matins

$E$ la messe e tout le servise

Ke fut dit en seinte eglise. [fol, 100a]

Tele vertue sa priere out

$104 \mathrm{Ke}$ homme ou femme pur ki priout

Ne faudra pas de grace trover,

Dunt vus orrez un cas cunter.

Il avint un jour issi

$108 \mathrm{Ke}$ une dame vint parler o li,

Tant cum fut a muster

$O$ li vint un esquier,

Queyntes e beaus e jolifs

112 A la dame fut avis

$\mathrm{Ke}$ trop fut a demesure,

Outrageus en porture!

„Vallet, dit ele, la mort vus gayte,

I16 Seez garny de sa deceyte,

Vus serrez deceu, s'il vus prent

En vostre folie sodeynement;

Nule rien est plus certeyne

$120 \mathrm{Ke}$ mort(e) ke prent e feble e seyn(e),

Ne rien n'est si noun certeyne

Cum est cel oure ke mettra la mayne.

Pernez garde la morte vus sut

I24 Pur vus prendre de jour ou nut

83 Deu 86 eus $87 \mathrm{Ke}$ ele enchesoun, teu 91 kanut $B$ tenir $W$ s. retenir 92 souzpir $95 \mathrm{ke}$ e, genz 96 lur, largemenz $97 \mathrm{ren}$, escoymousé $f$. Id 99. mout 100 coustume 105 faudreyt 109 mouster III beus II9 ren I2I ren 122 meyn I 24 v. mener 
E vus mener a joye ou peyne

Solum vostre fet demeyne.

S'il vus truve apparaysté,

128 A bone oure fustes né;

S'il vus truve desgarni,

Vus poez doter de estre hony."

Le vallet respondit: „En bonc fay,

132 Dame, priez dieu pur may;

Vus estes tenu une dame

De seintime vie e bone fame."

nSi voyllez ke pur vus prie,

136 Dit la dame, fetes ausie;

Alez devant cel auter,

Priez dieu de bon qüer,

$E$ jeo vus ayderai a mon poer

I40 Pur vus ausi de prier."

La dame en priers se mit,

E l'autre feseit, cum ele out dit.

En pou de oure il cria

144 nEydez, eydez, jeo mur ly ja! [fol. 100b]

Les damoyseles se hasterent

$\mathrm{Ke}$ o la duchesse illuc erent

Pur saver moun ke li deveit.

148 Il respondit e diseit

$\mathrm{Ke}$ tant sentit devocioun

Ke esteint serreit pas arsoun,

Si la dame ne cessat

152 De prier pur son estat.

A la dame est pus veneu

E devant li a tere cheheu:

„Dame, dit il, jeou vus pri

156 Pur Jhesu Crist, vostre amy,

Tel conseil me donez

$\mathrm{Ke}$ jeo puse estre sauvéz."

nAlez, dit ele, amy frer,

I60 A les frers precheours ke jeo ay cher;

$\mathrm{Si}$ entrez lur compaignie

$E$ amendrez vostre vie."

Li gentil homme de bon qüer

I64 Son consail fit sanz targer.

La seinte dame bien sentit

Par la grace del seint espirit

$\mathrm{Ke}$ humilité sur tut rien

126 Souloun 127 apparillée $129 f .2 a$ I3I respoundy, fey 132 deu, mey 134 seynte 137 avaunt 138 deu I4I prieres I44 murk 148 respount 153 venu 154 chehu 157 Teu 160 Les frere menours $\quad 161 f .26 \quad 163$ gentiz 164 connseil 165 ben 167 tote 
I68 A seinte alme fet plus de bien;

Pur ceo voleit honur despire

E subjeccion pur dieu elire.

Obedience vouha a un seint homme

I72 Ke mestre Conraud le livre nomme

De fere kanques il dirreit,

Hors pris le dette k'ele deveit

A son barun ki l'aout prise

I76 En la ley de seinte eglise.

Mestre Conraud li prodomme

La comanda de custume

De fere choses a li grevouses;

I 80 Mes ele feseit delicouses

Pur encrestre en ciel sa joye

Vers ou travail met homme en voie.

Mestre Conraud espia,

I84 Quele chose plus ama

E femme e juhel il la tolli

E remua bien loinz de li. [fol. $100 \mathrm{c}$ ]

Ele soleit dire a ses aunceles:

I88 „Oyer ne purrey meliour noveles

Ke povre ne fuse devant ma mort

$\mathrm{Ke}$ ceo me serreit grant comfort."

Dunt ele soleit acune fez

192 Les dras vestir detirez

En sa chambre privement

$\mathrm{E}$ dire as autres joyousement:

„Issi irrey un de ces jours

196 Entre povres pur dieu amours."

Chescune nute, devant le jour

Ele leva de son seignour

$\mathrm{Ki}$ pur sa bounte la suffri

$200 \mathrm{De}$ fere ceo ke plut a li;

E se mit en oreyson

En le oratorie de sa meson.

Une garcette assingneit,

$204 \mathrm{Si}$ par tens ne aveylleit

$\mathrm{Ke}$ cle li tochat le pée

Par unt ele fut aveillée.

La garcette vint une nut(e)

208 De fere ceo ke assingnèe fut(e),

Avint issi k'ele tochaa

Les pez son segnour ele aveylaa

Li grant sire dit sanz desray:

2 I2 "Ore sus, dame, priez pur may."

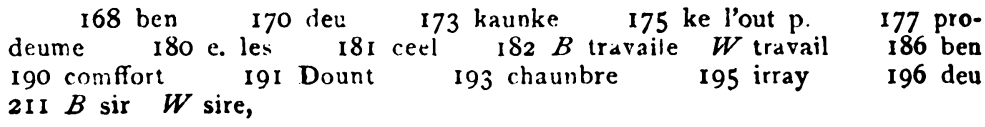


Tant fut la dame de ben port

$\mathrm{Ke}$ chescun prodomme out confort

La bone dame de visiter

216 Pur sa manere aviser.

Avint issi ke un grant segnour,

Un count la vint ver un jour:

$E$ fu maunde k'ele venit,

220 Mes quoyntement se escundit.

Rien ne valut sa reson

Ke li sires de la meson

Si la manda k'ele venit

224 Al grant segnour ki la attendit.

Ele remanda a son segnour

K'ele vendreit sanz long demour,

Ele out donée avant cel oure

228 A povres tant de sa vesteure

$\mathrm{Ke}$ povrement esteit vestue

Pur son segnour trop se ennue

A genuz se mit en plorant

232 E dieu pria tut pussant

$\mathrm{Ke}$ a li mustra dunc sa grace.

$E$ meyntenant devant sa face

Un angel apparut o vesture

236 De mult noble atiffure:

${ }_{n}$ Dame, dit il, sanz targer

Ore vus facez atirer

$E$ fetes le comandement

240 De vostre segnour sanz attent."

La dame descendit meyntenant

E apparut si lusant

Ke ne i a homme en la sale

$244 \mathrm{Ke}$ de merveyle ne esteit pale.

Tant se sunt en merveyléz

Ke pour e joye sunt entremelléz

Mult courtement se aquita

248 E en sa chambre returna;

Lors se aforcea de plus amer

Jesu Crist e honurer.

De hodivesce n'out ke fere

252 En chescun tens vout louer quere,

En priers estre devant manger,

Visiter malades apres manger

Cosut les dras de povres genz

256 De les eglises les vestimenz

Autre travaylles ne vout user,

214 prodoun, counfort 220 escoundit 
Kar ele entendi de plus gaygner;

Son cors pena par juner

260 E ses oyez par mult veiller.

La parole ne fut pas veyne,

Mes de seinteté tute pleyne;

Kant ahura le sacrement,

264 Ahoura si devoutement

Ke plusurs fez li fut mustrée

Grant merveille en privetée.

Dount une fez devant le auter

268 Tant resceut de joye en qüer

$\mathrm{Ke}$ ele fut ausi cum ravye;

Une damoisele en ki se affie [fol. ror a]

Pus apres de li enquit,

272 Quele merveile illuc vit.

Ele respondit meyntenant

$\mathrm{Ke}$ ja ne dirreit a homme vivant

Sa mere morte la apparut

276 En avision une nut;

Si l'ad dit en tele manere:

${ }_{n}$ Bele fille, jeo su ta mere;

Pur celes anguses ke o vus senti,

280 Eez ore de moy merci.

Jeo su, fille, dure maignée

En purgatore pur mon pechée."

I.a seinte dame leva del lit

284 E ses genuz a tere mit,

Plurut sur dieu mult tendrement;

Ele pria de tutte sa entent

Pur sa seinte passion

288 Ke merci ut de ceo prison.

Ke sa priere out vertué,

En poy de oure fu bien conué

Ke autre fez meme la nut(e)

292 Bele e clere la appirut(e).

La mercia de tel secour

E dit: "Loée seit mon creatour!

Jeo su quite de chescune peyne

296 E me voys a joye certeyne."

Un jour vint en l'ospital,

Trova un homme ki out tel mal

Ke muhet fut, paralitik,

300 Ne mover se pout hors de lit.

La dame ne sout dunt esteit,

260 s. eux, mout 273 respundi 275 le $277 \mathrm{Si}$ la d. teu 28 I dur marniée 282 purgorie, 285 deu 288 ceu $289 B$ vertuée, $W$ vertue $f \cdot 3^{b} . \quad 290$ ben 292 le 293 teu 298 teu 
Vout saver, mes rien ne parleit;

E lu de parole hocha sa teste,

304 La dame vit par tele geste

Ke la parole out perdu:

nJeo vus comand en noun Jesu,

Dit la dame, ke of moy parlez

308 E del lit seyn sus levez!"

Sanz plus demorer sein leva

E O la dame dunc parla;

Si l'ad dit, ke pus fut prové(e)

312 Ke unk mes ne out parlé(e) [fol. ror b]

E meyntenant la dame s'en fut

Kant tel miracle apparceut;

Cele ke fut de dieu privée

3 I6 Ne vout estre de homme priesé(e).

La bone dame ad prechéz

Son segnour plusurs fez

En la tere seinte de aler

320 Pur dieu servir e ceel aver.

Ly noble duk son conseil crat,

La se mit et la morut.

I.es uns de sa parentée,

324 Kel la dame unt poy amée

Mistrent sure a la dame

Destruction e male fame.

Si la unt engetté del pays

328 E tuz ses biens de li pris.

La seinte dame s'en ala

$O$ une servante k'of li demora;

Une povre femme encountra

$33^{2}$ A qui sustenance avant dona

Ke fit la dam cheher enclin,

Ne la vout lesser le chemin.

La dame leve e se escut

336 Ne aveit de ces fors dedut;

Mult fut joyeuse de sa poverté

Kar dieu la fit de joye certé.

Ele vit un jour en le devant

340 Ja damoysele regardant

Vers mount au ceel bien longment

Comence a riere joyeusement

$E$ pus apres en poy de oure

344 Comence plorer a demesure,

302 ren $303 \mathrm{En} \quad 310$ dounk 3 II Si la d. 314 teu 315 deu $320 \mathrm{deu}$, cel $325 \mathrm{~s}$. en i. 326 Destrucioun 327 ie 332 souste. naunce 337 Mout $338 \mathrm{deu} 34 \mathrm{I}$ ben loungement 
E meyntenant derichef

Comence rire e lever le chef

$E$ dit issi tut en haut:

348 "Certes, sir, il me ne chaut

Quele dolur ke me reygne,

Mes ke vestre promesse teygne."

La damoysele se merveila

$352 \mathrm{Ke}$ la chere si chaungea

Ore en risée, ore en plour

Si la pria par grant douzour

K'ele vousit la veritée

356 Dire de chosee ke fut mustrée.

„Volentiers, dit ele, par issi

$\mathrm{Ke}$ vus ne le diez a nuli.

Kant jeo regardey al fermament

360 Jeo vy Jhesu appertement;

De fin joye comenceay rire,

Mes le semblant tost me empire,

Kant se retret si sodeynement

364 De ceo estei jeo mult dolent.

Autre fez jeo comenceay rire,

Kant celi en ki le ceel se mire

Sa doulce presence me mustra

368 E doulcement vers moy parla

E dit: Si vas volez tenir a moy,

Jen voil tuz jours estre o toy.

$E$ jeo respondi en la manere

372 Cum vus oytes o lee, chere."

Ele vint un jour encontrant

Genz ki amenerent un enfant

$\mathrm{Ki}$ pus le tens ke nasquit

376 Unke rien des oiz ne vit.

Il ne aveit oil dunt poeit ver

En lu des oiz ni out fors pel.

La dame out pitée de la veue

380 E de pitée dunc esmue

Tocha la face de la mayn desire

En cel leu ou le oil dut estre;

E par dieu resscut l'enfant

384 Deuz oiz clers e lusanz.

Kant la novele de sa poverté

Al rey de Ungarie son pere fu contté

Par ses chivalers fit maunder

388 Pur quere sa fille e a li mener.

$346 B$ rier $W$ rire $353 f \cdot 3^{d} \quad 354 B$ dulceur, $W$ douzour 364 mout 368 doucemen: $376 \mathrm{ren}$, euz 377 a. huyi $378 \mathrm{~d}$. cuz 380 dounk 381 du meyn 384 euz, lusaunz 385 fol. $4 a \quad 386$ t. certe 
Un cunt ki fut chef messager,

Kant trova la dame sere e filer:

"Coment, dit il, ke est ces ke vey?

392 Estes vus dame e fille au rey?"

Il la rout o li amener,

Mes ele ne vout en nule mener.

Ses amys la vunt purchacer

396 En leu de doner grant aver; [fol. IOId]

Ja si tost le tresor ne out

$\mathrm{Ke}$ a povrez genz ne donout.

En bref tens apres cel oure

400 Les ossemenz son segnour

En pays sunt reportez

Ou la dame s'en est alez;

$E$ fit le honour ke ele pout,

404 E pus apres s'en alout

En privée leu pur dieu servir,

Si out dunc son desir.

Ele mena mult seinte vie

408 E cele vie ad bien chevie.

La nute ke morust fut oye

Sus la meson grant melodye

De plusurs oysels ke angels esteient

$412 \mathrm{Ke}$ son espirit al ciel porteient.

Jeo pri la dame pur dieu amour

Ke vers dieu nus seit en socour. Amen.

$394 B$ manere, $W$ maner 401 sount 405 deu 407 mout 408 ben 4 II oyseus 4 I 2 esperit au ceel 4 I 3 deu 414 deu $B$ s. s. $W$ s. en s.

LOUIS KAKL. 